\title{
Kondo screening cloud in a one dimensional wire: Numerical renormalization group study
}

\author{
László Borda \\ Department of Theoretical Physics and Research Group "Theory of Condensed Matter" of the Hungarian Academy of Sciences, \\ Budapest University of Technology and Economics, Budafoki út 8. H-1521 Budapest, Hungary
}

(Dated: June 25, 2018)

\begin{abstract}
We study the Kondo model - a magnetic impurity coupled to a one dimensional wire via exchange coupling- by using Wilson's numerical renormalization group (NRG) technique. By applying an approach similar to which was used to compute the two impurity problem we managed to improve the bad spatial resolution of the numerical renormalization group method. In this way we have calculated the impurity spin - conduction electron spin correlation function which is a measure of the Kondo compensation cloud whose existence has been a long standing problem in solid state physics. We also present results on the temperature dependence of the Kondo correlations.

PACS numbers: 72.15.Qm, 73.63.Kv
\end{abstract}

Introduction - As being one of the most interesting quantum impurity problems, the Kondo effect[1] - when a localized impurity spin interacts with the itinerant electrons via spin exchange coupling- has been studied for decades both theoretically and experimentally [2] and has recently come to its renaissance when it has been predicted to appear [3] and observed [4] in quantum dot systems. Even though the Kondo effect seems to be wellunderstood now, still, there is a controversial question which has not been clarified yet: Whether there exists an extended "Kondo screening cloud" or not?

In case of the single channel Kondo problem -when the local spin is coupled to one band of conduction electronsthe interaction with the electrons lifts the ground state degeneracy of the isolated spin resulting in an effective "screening" of the impurity spin below a certain energy scale, the so-called Kondo temperature $T_{K}$. It is adequate to ask the question what is the typical length scale in which the impurity spin gets screened. As a simple estimate, just by comparing the scales of the competing kinetic and binding energies, one easily gets a length scale, the so-called Kondo coherence length, $\xi_{K}=\hbar v_{F} / k_{B} T_{K}$. By substituting the typical Fermi velocity $v_{F}$ in metals, and taking a typical Kondo temperature $T_{K} \sim 1 K$, one gets $\xi_{K} \sim 1 \mu m$, which is comparable with, or even larger than the typical dimension of today's mesoscopic devices. However, to measure that screening cloud is highly nontrivial from experimental point of view.

In order to observe the screening cloud, one has to measure correlations between the impurity spin and the conduction electron spin density. It is very difficult to imagine such kind of a measurement in bulk metallic samples. Recently, it has become possible to perform scanning tunneling microscopy (STM) measurements on metallic surfaces addressing a single magnetic impurity and its close neighborhood [5]. In those experiments the screening cloud was not observed since the STM tip probes the local charge density of states which is affected by the Kondo correlations in the close neighborhood of the im- purity only. Even in case of having spin-polarized STM tips, it is very difficult to measure the spin correlations since the Kondo effect manifests itself in continuous, coherent spin flip processes at a time scale $\sim 1 / T_{K}$ meaning that one has to measure at a frequency of tens of gigahertzs. The idea of suppressing the impurity spin fluctuations by applying a local magnetic field is doomed to fail since a magnetic field which is large enough to suppress the spin fluctuations is necessarily large enough to destroy Kondo correlations as well.

There were experimental setups proposed in order to measure the Kondo cloud in confined systems[6], e.g. in case of a quantum dot attached to one dimensional lead with length shorter than, or comparable with $\xi_{K}$. Such kind of a proposal suffers from the facts that it is destructive, i.e. the Kondo effect disappears for one $d i$ mensional leads shorter than $\xi_{K}$ and one can still argue that the suppression of the Kondo effect is a result of having the level spacing in the lead $\delta \epsilon>T_{K}$ for short enough leads therefore one does not need the theoretical construction of the screening cloud to explain the experimental findings. In case of an impurity embedded into a higher dimensional environment, another length scale $l_{K}$ emerges [7] by equating $\Delta\left(l_{K}\right)=T_{K}$ where $\Delta\left(l_{K}\right)$ stands for the mean level spacing in a box with size $l_{K}$. For the case of $D \geq 2$ that length scale can be substantially smaller than $\xi_{K}$. In 1D systems, which are in the focus of the present paper, the two length scales are essentially equal.

Despite those challenges, there were proposals published to observe the Kondo screening cloud. These proposals deal with the Knight shift [8], persistent current [9] or conductance of mesoscopic systems [10].

Very recently, Hand and his coworkers established a proportionality between the weight of Kondo resonance and the spatial extension of the Kondo correlations in mesoscopic systems [1]. This fact allows another, spectroscopic way to observe the Kondo screening cloud. Those proposals are very promising even though the ex- 
perimental realizations are yet to come.

In the present paper we are less ambitious since our goal is not to propose new ways of observation but to extend the powerful and numerically exact NRG[12] to be capable to compute spatial correlations and therefore make predictions for the outcome of future experiments.

Model and definition of correlation functions - The Hamiltonian of the Kondo model can be written as

$$
H=J \vec{S} \vec{\sigma}(0)+\sum_{k, \mu} \varepsilon_{k} c_{k \mu}^{\dagger} c_{k \mu}
$$

where $\vec{S}$ is the impurity spin $(S=1 / 2$, located at the origin), $c_{k \mu}^{\dagger}\left(c_{k \mu}\right)$ creates (annihilates) a conduction electron with momentum $k$ and spin $\mu$, while $\vec{\sigma}(0)=\psi^{\dagger}(0) \vec{\sigma} \psi(0)$ stands for the electron spin density at the position of the impurity. As it is mentioned above, the system described by Eq.(11) forms a singlet below the Kondo temperature $T_{K} \sim D_{0} \exp \left(-1 / 2 \varrho_{0} J\right)$, where $D_{0}$ is the high energy cutoff and $\varrho_{0}$ is the density of states of the conduction electrons. (For sake of simplicity, we consider a band with a constant density of states in the following. Moreover, we restrict ourselves for $1 \mathrm{D}$ electrons because it is easier to treat them numerically. When it is adequate, we will comment the case of higher dimensions as well.) A quantity which measures the spatial extension of the singlet is the equal-time correlator of the impurity spin and conduction electron spin density at position $x$ :

$$
\chi_{t=0}(x)=\langle\vec{S} \vec{\sigma}(x)\rangle_{t=0} .
$$

Since we know that the ground state of the Hamiltonian Eq.(11) is a singlet, we can easily derive a sum rule for the equal time correlator at zero temperature:

$$
\int_{0}^{\infty}\langle\vec{S} \vec{\sigma}(x)\rangle_{t=0} \mathrm{dx}=-\frac{3}{4} .
$$

Method - To calculate spatial correlations is a nontrivial task since most of the methods used to investigate the Kondo model are not able to reproduce correlation functions. Comparatively only a very little of the theoretical study has been focused on spatial correlations: Perturbative calculations have been performed [13, 14] as well as Monte Carlo analysis [15]. In the present paper we extend NRG to compute the Kondo correlations.

Wilson's NRG -the most useful numerically exact method to obtain correlation functions [16]- suffers from the very bad spatial resolution away from the impurity. This fact is a direct consequence of the cornerstone of the method, the logarithmic discretization of the conduction band. In Wilson's NRG technique one introduces a discretization parameter, $\Lambda$ and with the help of that discretizes the conduction band logarithmically by dividing it into intervals (i.e. the $n$th interval is ] $\left.-D_{0} \Lambda^{-n} ;-D_{0} \Lambda^{-n-1}\right]$ for negative, and

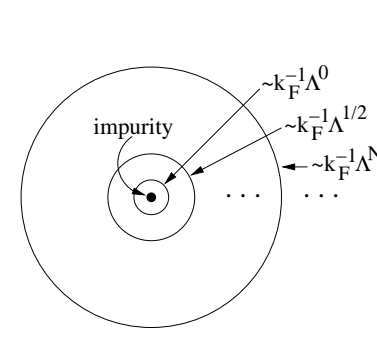

a

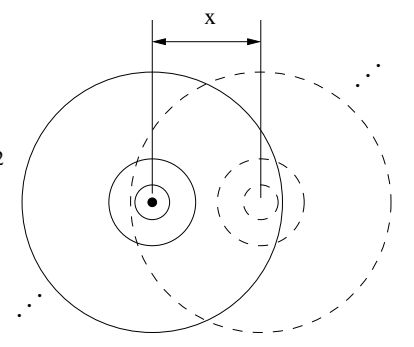

b
FIG. 1: (a) Spherical shells in real space depicting the extents of wave functions of states which are represented as on-site states on the Wilson chain. As shown, the NRG method has a good spatial resolution around the impurity only. (b) A straightforward extension of NRG tackles that problem providing a good spatial resolution at the impurity site as well as at another, freely chosen point by introducing a second electron field.

$\left[D_{0} \Lambda^{-n-1} ; D_{0} \Lambda^{-n}\right.$ [ for positive energies measured from the Fermi energy and $D_{0}$ stands for the half bandwidth) and keeping one mode per interval only. As a next step, one maps the problem onto a semi-infinite chain with the impurity at the end by means of a Lánczostransformation. As a consequence of the logarithmic discretization, the hopping amplitude falls off exponentially along the chain allowing us to diagonalize the problem iteratively. As it is shown in Ref.[12] the states represented as on-site states on the Wilson-chain correspond to extended states in real space with a typical spatial extension

$$
r_{N} \sim \frac{1}{k_{F}} \Lambda^{N / 2},
$$

where $N$ is the site index along the chain. It is obvious that the numerical renormalization group method has a good spatial resolution at the position of the impurity only. [See Fig 1a.]

To tackle that problem, we introduce two electron fields, one is located at the impurity site, $\psi_{0}=\psi(0)$ and another one at point $x$ where we want to evaluate the correlation function, $\psi_{1}=\psi(x)$. [See Fig.10.] (A very similar procedure was applied in the numerical renormalization group treatment of the two impurity problem[17].) Of course, these fields are not orthogonal, i.e. they do not fulfill the canonical anticommutational relations. As a next step, we can introduce the proper linear combinations of these fields,

$$
\psi_{ \pm}=1 / \sqrt{2}\left(\psi_{0} \pm \psi_{1}\right)
$$

which are now anticommuting but the corresponding density of states is modified i.e. it acquires energy dependence, e.g. for $1 \mathrm{D}$ electrons $\varrho_{ \pm}^{1 D}(\varepsilon)=\varrho_{0} / 2[1 \pm$ $\left.\cos \left(\varepsilon x / v_{F}\right)\right]$. (In $2 \mathrm{D}$ the density of states reads $\varrho_{ \pm}^{2 D}(\varepsilon)=$ $\varrho_{0} / 2\left[1 \pm J_{0}\left(\varepsilon r / v_{F}\right)\right]$ where $J_{0}$ is the zeroth Bessel function, while in $3 \mathrm{D} \varrho_{ \pm}^{3 D}(\varepsilon)=\varrho_{0} / 2\left[1 \pm \sin \left(\varepsilon r / v_{F}\right) /\left(\varepsilon r / v_{F}\right)\right]$. 


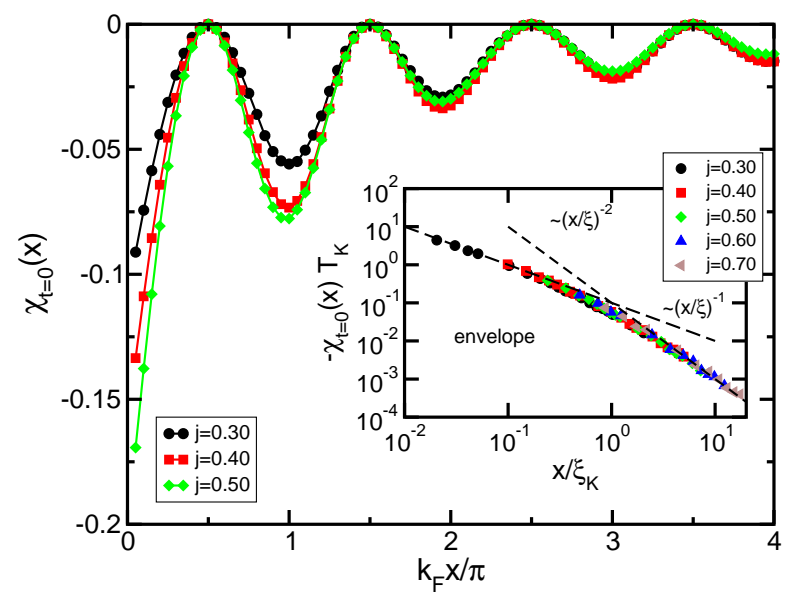

FIG. 2: (Color online) The equal time spin-spin correlation function, $\chi_{\mathrm{t}=0}(x)=\langle\vec{S} \vec{\sigma}(x)\rangle$ as a function of the distance $x$ measured from the impurity for different values of the Kondo coupling $j=\varrho_{0} J . \quad \chi_{\mathrm{t}=0}(x)$ oscillates as $\sim \cos ^{2}\left(k_{F} x\right)$. As shown in the inset the envelope of the oscillating part (i.e. $\chi_{\mathrm{t}=0}\left(x=n \pi / k_{F}\right)$ where $n$ is a integer) crosses over from $\sim x^{-1}$ to $\sim x^{-2}$ at around the Kondo coherence length, $\xi_{K}$. The envelope function for different couplings nicely collapse into one universal curve.

As we see, the oscillations of the even/odd density of states decay as $\sim r^{-(D-1) / 2}$, where $D$ is the dimensionality of the problem.) In the new basis the Kondo Hamiltonian reads

$$
\begin{aligned}
H & =\frac{1}{2} J \vec{S}\left(\psi_{+}^{\dagger}+\psi_{-}^{\dagger}\right) \vec{\sigma}\left(\psi_{+}+\psi_{-}\right) \\
& +\sum_{i= \pm \mu=\uparrow, \downarrow} \int d \varepsilon \varrho_{i}(\varepsilon) \varepsilon c_{i \varepsilon \mu}^{\dagger} c_{i \varepsilon \mu} .
\end{aligned}
$$

In this representation $\vec{\sigma}(x)=1 / 2\left[\psi_{+}^{\dagger}-\psi_{-}^{\dagger}\right] \vec{\sigma}\left[\psi_{+}-\psi_{-}\right]$ is represented in a high accuracy. In brief, the main idea behind the above transformation is that one can get a very good spatial resolution at the impurity site as well as at another freely chosen position $x$ if one is willing to pay the price of (a) having two electron channels mixed by the interaction, (b) having energy dependent density of states and (c) having a separate NRG iteration for every different $x$ values. Those difficulties are possible to be handled: The most serious one amongst them is the extension of NRG to arbitrary density of states which has been solved by Bulla et al. 18]

Results - In this NRG scheme the calculation of the equal time correlation function is rather simple: $\chi_{t=0}(x)$ appears to be a static thermodynamic quantity which can be evaluated with a high precision. The results for different Kondo couplings are shown in Fig[2] the correlation function oscillates as $\sim \cos ^{2}\left(k_{F} x\right)$. As it is shown in the inset of Fig, 2, the envelope of the correlation function for different Kondo couplings nicely collapse into one universal curve (apart from the points $x \leq \pi / k_{F}$ which show non-universal, coupling-dependent behavior, not plotted

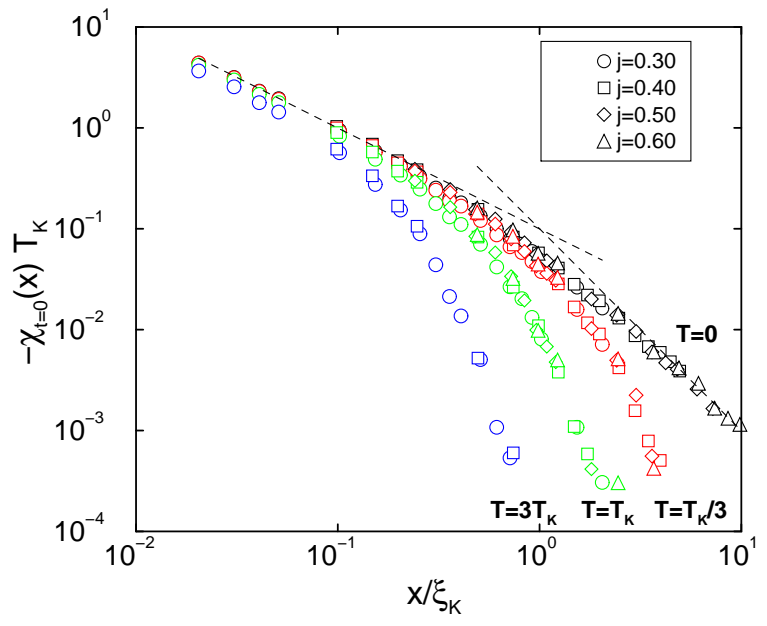

FIG. 3: (Color online) The envelope of $\chi_{t=0}(x)$ for different couplings and different temperatures: Any finite temperature introduces another energy scale, $\xi_{T}=\hbar v_{F} / k_{B} T$ at which the envelope function crosses over from an algebraic to an exponential decay.

in the inset). The envelope curve (i.e. $\chi_{\mathrm{t}=0}\left(x=n \pi / k_{F}\right)$ where $n$ is an integer) changes its decay from $\sim 1 / x$ to $\sim 1 / x^{2}$ at around $\xi_{K}$. Our results supports the heuristic picture of the Kondo ground state: The impurity is locked into a singlet with an electron whose wave function is spread out over a distance of $\xi_{K}$. If we test the correlations on a length scale much shorter than $\xi_{K}$ the impurity appears to be unscreened and a perturbative analysis can be performed 14] yielding $\chi_{\mathrm{t}=0} \sim 1 / x$. On the other hand, the impurity is screened for distances $x \gg \xi_{K}$ and Nozières' Fermi liquid theory applies which predicts $\sim 1 / x^{2}$ decay in $1 \mathrm{D}[19]$. There is, however, a rather wide crossover regime for which there is no reliable analytic method of calculating $\chi_{\mathrm{t}=0}(x)$. From perturbative aspect this is the region where the logarithmic corrections to $\sim 1 / x$ becomes large 14]. Alternatively, following the argumentation of the authors of Ref 11 , this is the region where the impurity can be described by a fluctuating spin resulting in exponential decay which is overriden by the $\sim 1 / x^{2}$ decay for even larger distances.

To plot the universal curve in the inset of Fig 2 we have chosen the definition of $T_{K}$ based on the NRG flow of the energy levels: We identified $T_{K}$ as the energy scale at which the energy of the first excited state reaches $80 \%$ of its fixed point value. We have taken this definition since the energy spectrum is the most basic and most reliable result of any NRG calculation and -as a consequence of the universality of the Kondo effect- the value of $T_{K}$ obtained in this way can differ from that of other definitions up to a constant multiplying factor only.

Note that the results shown in Fig 2 correspond to 1D: in the case of higher dimensions the correlation function tends to zero even faster since the sum rule (Eq $\sqrt[3]{3})$ fixes 
its integral. This fact enters the numerical calculation through the $\sim r^{-(D-1) / 2}$ decay of density of states oscillations and makes the calculation of $\chi_{t=0}(x)$ challenging for dimensions higher than $D=1$.

Up to this point we have considered the case of zero temperature $T=0$. However, it is known that the temperature plays an essential role in Kondo physics: Kondo correlations are destroyed when the temperature reaches the Kondo scale $T \sim T_{K}$. It is adequate to ask the question how does this fact show up in $\chi_{t=0}(x)$ ?

Since $\chi_{t=0}(x)$ is a static quantity we obtain its complete temperature dependence by numerical renormalization as the iteration proceeds. In Fig 3 we show the results for $T=0, T=T_{K} / 3, T=T_{K}$, and $T=3 T_{K}$. As it is transparent from the curves, the effect of finite temperature shows up in the appearance of another length scale, the thermal length scale, $\xi_{T}=\hbar v_{F} / k_{B} T$. For $x<\xi_{T}$ the correlation function $\chi_{t=0}(x)$ is not much affected while for $x>\xi_{T}$ the correlations are cut off exponentially.

Based on those results we can now interpret the role of finite temperature in Kondo screening as follows: The fact that the impurity spin is perfectly screened at $T=0$ is reflected in the sum rule given by Eq.(3). At any finite temperature the integral is reduced and can approximately rewritten as $\int_{0}^{\xi_{T}} \chi_{t=0}(x) d x$. When the temperature is lower than the Kondo temperature $T<T_{K}$, the corresponding thermal length scale is larger than $\xi_{K}$ Since $\chi_{t=0}(x)$ has its most weight in the region $x<\xi_{K}$, the effect of a small temperature is just a small correction to the perfect screening obtained at $T=0$. Given that in the $x>\xi_{K}$ regime $\chi_{t=0}(x) \sim x^{-2}$, the correction appears to be linear in $T$. In contrast, for $T>T_{K}$ the corresponding $\xi_{T}$ is shorter than $\xi_{K}$ and the correction to the integral is not small and consequently, we cannot speak about the screening of the local moment any more.

Conclusions - We have shown that Wilson's NRG technique is capable to handle spatial correlations if we apply a straightforward extension. To demonstrate that we have computed the $\langle\vec{S} \vec{\sigma}(x)\rangle_{t=0}$ correlator for a one dimensional Kondo system and shown that the decay of spin correlations crosses over from $\sim x^{-1}$ to $\sim x^{-2}$ at around the Kondo coherence length, $\xi_{K}=\hbar v_{F} / k_{B} T_{K}$. We have shown by calculating the temperature dependence that any finite temperature introduces a new energy scale beyond which the Kondo correlations vanish exponentially. Our method is -apart from the numerical challenges- easy to generalize to other impurity models. A very attractive example would be the two channel Kondo model to see how the non-Fermi liquid nature of the ground state shows up in the spatial spin correlations.

The author acknowledges the fruitful discussions with G. Zaránd, R. Bulla, D. Goldhaber-Gordon, A. Zawadowski and O. Újsághy. This work was supported by EC RTN2-2001-00440 "Spintronics", Projects OTKA D048665, T048782, T046303 and by the János Bolyai Scholarship.
[1] J. Kondo, Prog. Theor. Phys. 32, 37 (1964).

[2] For a review see A.C Hewson, The Kondo Problem to Heavy Fermions, Cambridge University Press (1993).

[3] L.I. Glazman and M.E. Raikh, JETP Lett. 47, 452 (1988); T.K. Ng and P.A. Lee, Phys. Rev. Lett. 61, 1768 (1988).

[4] D. Goldhaber-Gordon, H. Shtrikman, D. Mahalu, D. Abusch-Magder, U. Meirav, and M.A. Kastner, Nature (London) 391, 156 (1998); S.M. Cronenwett, T.H. Oosterkamp, and L.P. Kouwenhoven, Science 281, 540 (1998); J. Schmid, J. Weis, K. Eberl, and K. von Klitzing, Physica (Amsterdam) 256B-258B, 182 (1998); W.G. van der Wiel, S. De Franceschi, T. Fujisawa, J.M. Elzerman, S. Tarucha, and L.P. Kouwenhoven, Science 289, 2105 (2000).

[5] J. Li, W.-D. Schneider, R. Berndt, and B. Delley, Phys. Rev. Lett. 80, 2893 (1998); V. Madhavan, W. Chen, T. Jamneala, M. F. Crommie, and N. S. Wingreen, Science 280, 567 (1998); H. C. Manoharan, C. P. Lutz, and D. M. Eigler, Nature (London) 403, 512 (2000).

[6] P. S. Cornaglia and C. A. Balseiro, Phys. Rev. B 66, 115303 (2002); P. S. Cornaglia and C. A. Balseiro, Phys. Rev. B 66, 174404 (2002).

[7] W. B. Thimm, J. Kroha, and J. v. Delft, Phys. Rev. Lett. 82, 2143 (1999).

[8] E. S. Sorensen and I. Affleck, Phys. Rev. B 53, 9153 (1996); J. P. Boyce and C. P. Slichter, Phys. Rev. Lett. 32, 61 (1974); Phys. Rev. B 13, 379 (1976); K. Chen, C. Jayaprakash, and H. R. Krishna-Murthy, Phys. Rev. Lett. 58, 929 (1987).

[9] I. Affleck and P. Simon, Phys. Rev. Lett. 86, 2854 (2001); ibid 88, 139701 (2002); H. P. Eckle, H. Johannesson, and C. A. Stafford, Phys. Rev. Lett. 87, 016602 (2001); ibid 88, 139702 (2002); P. Simon and I. Affleck, Phys. Rev. B 64, 085308 (2001); E. S. Sorensen and I. Affleck, Phys. Rev. Lett. 94, 086601 (2005).

[10] P. Simon and I. Affleck, Phys. Rev. Lett. 89, 206602 (2002).

[11] T. Hand, J. Kroha, and H. Monien, Phys. Rev. Lett. 97, 136604 (2006).

[12] K.G. Wilson, Rev. Mod. Phys. 47, 773 (1975); H.R. Krishna-murthy, J. W. Wilkins, and K. G. Wilson Phys. Rev. B 21, 1003 (1980); Phys. Rev. B 21, 1044 (1980).

[13] R. H. Bressmann and M. Bailyn, Phys. Rev. 154, 471 (1967); M. S. Fullenbaum and D. S. Falk, Phys. Rev. 157, 452 (1967); H. Keiter, Z. Phys. 223, 289 (1969);J. Gan, J. Phys. Condens. Matter 6, 4547 (1994)

[14] V. Barzykin and I. Affleck, Phys. Rev. B 57, 432 (1998).

[15] J. E. Gubernatis, J. E. Hirsch, and D. J. Scalapino, Phys. Rev. B 35, 8478 (1987).

[16] T. A. Costi, A. C. Hewson, and V. Zlatic, J. Phys.: Condens. Matter 6, 2519 (1994).

[17] B. A. Jones, C. M. Varma, and J. W. Wilkins, Phys. Rev. Lett. 61, 125 (1988); J. B. Silva, W. L. C. Lima, W. C. Oliveira, J. L. N. Mello, L. N. Oliveira, and J. W. Wilkins Phys. Rev. Lett. 76, 275 (1996)

[18] R. Bulla, Th. Pruschke, A.C. Hewson, J. Phys.: Condens. Matter 9, 10463 (1997); C. Gonzalez-Buxton and K. Ingersent, Phys. Rev. B 57, 14254 (1998); K. Chen and C. Jayaprakash, Phys. Rev. B 52, 14436 (1995).

[19] H. Ishii, J. Low Temp. Phys. 32, 457 (1978). 